\title{
Viscosity Measurement Using Optical Tracking of Free Fall in Newtonian Fluid
}

\author{
N. KhelOUFI ${ }^{a, b, *}$ AND M. LOUNIS ${ }^{b, c}$ \\ ${ }^{a}$ Laboratoire de Géodésie Spatiale, Centre des Techniques Spatiales, \\ BP 13 rue de Palestine Arzew 31200 Oran, Algeria \\ ${ }^{b}$ Laboratoire LAAR - Faculté de Physique, Département de Technologie des Materiaux, \\ Université des Sciences et de la Technologie d'Oran USTO-MB, BP 1505, El M'Naouer 31000 Oran, Algeria \\ ${ }^{c}$ Faculté des Sciences et de la Technologie, Université de Khemis Miliana - UKM, \\ Route de Theniet El Had 44225 Khemis Miliana, Algeria \\ (Received July 1, 2014; revised version April 6, 2015; in final form May 6, 2015) \\ This paper presents a novel method in viscosity assessment using a tracking of the ball moving in Newtonian \\ fluid. The movement of the ball is assimilated to a free fall within a tube containing liquid of whose we want \\ to measure a viscosity. In classical measurement, height of fall is estimated directly by footage where accuracy \\ is not really considered. Falling ball viscometers have shown, on the one hand, a limit in the ball falling height \\ measuring, on the other hand, a limit in the accuracy estimation of velocity and therefore a weak precision on the \\ viscosity calculation of the fluids. Our technique consist to measure the fall height by taking video sequences of the \\ ball during its fall and thus estimate its terminal velocity which is an important parameter for cinematic velocity \\ computing, using the Stokes formalism. The time of fall is estimated by cumulating time laps between successive \\ video sequences which mean that we can finally estimate the cinematic viscosity of the studied fluid.
}

DOI: 10.12693 /APhysPolA.128.123

PACS: 83.85.Jn, 83.60.Fg

\section{Introduction}

Viscosity is known as a material (fluid) resistant to the movement between layers under shear stress action, and characterizes its suitability to flow under internal forces such as gravity. It is due to the friction between neighboring particles in a fluid that are moving at different velocities [1]. When the fluid is forced through a tube, the fluid generally moves faster near the axis and very slowly near the walls. Therefore, some stress (such as a pressure difference between the two ends of the tube) is provided by the gravity forces and is needed to overcome the friction between layers and keep the fluid moving. For the same velocity pattern, the stress required is proportional to the fluid's viscosity.

Because studying a flow of viscous matters is sometimes fastidious and requires complicated disposition, we can, as the movement is relative in Eulerian reference, change the stream, otherwise, instead to force the fluid to move, we make the obstacle moving around the fluid and then study its movement using the Navier-Stokes equations and fluid cinematic formalism [2].

That is why many viscosity measurements based on Stokes or Hoppler [3] methods take into consideration a falling ball within cylinder tube filled up by the studied substance, and estimate its terminal velocity indispensable for above cited methods to compute the cinematic viscosity in $\mathrm{mm}^{2} / \mathrm{s}$.

*corresponding author; e-mail: dinori2014@gmail.com
The novelty in this paper is the technique by which the distance browsed by the falling ball is estimated relief of optical tracking, based on video capture of movement.

For this purpose, an experimental arrangement has been elaborated and tested. More details are available in next paragraph.

\section{Methodology}

\subsection{Experimental}

In order to perform the experiments, a set of different diameters balls made with different materials with different density such as steel (7.8), Altuglass PMMA (1.3) and oak wood (0.8) [4] have been used.

Every material has its proper characteristics such as density $\rho$ and viscous friction factor given by the Stokes law as $k=3 \pi D$, where $D$ is the ball diameter $[5,6]$. These parameters are determined for ball behavior in the fluid and for viscosity calculation accuracy using the most popular two models adopted in this work. Two industrial (SAE140, SAE40) and vegetal fluids (olive oil) in different temperatures varying by the step of $20^{\circ} \mathrm{C}$ (range of $19.7^{\circ} \mathrm{C}$ to $98.6^{\circ} \mathrm{C}$ ) are tested. We retain that fluids in this work are Newtonian and transparent for the need of optical aspect of the measures which request a digital high quality camera and high visibility of the ball in its fall within the translucent vertical nozzle. Placed in front of the tube using an adequate system which allow good lightening and good object plan distance, the camera is synchronized with the beginning of the fall. Lightening conditions are crucial for the visibility and the quality of images to be treated in order to avoid artifacts which 
can blemish the results in order to have necessary contrast needed in the images preprocessing phase.

Finally a synchronous timing between ball release and video capture launching is ensured for good estimation of the fall height.

\subsection{Data processing}

The fluid flow phenomena around the ball are governed by the Navier-Stokes equation for laminar regime as follows:

$$
\begin{aligned}
& \rho \frac{\partial v}{\partial t}-\nabla \eta\left(\nabla v+(\nabla v)^{\mathrm{T}}\right)+\rho v \nabla v+\nabla p=F, \\
& \nabla v=0,
\end{aligned}
$$

in which $\rho$ is the density of fluid, $\eta$ - its viscosity in $\mathrm{N} \mathrm{s} / \mathrm{m}^{2}, \nu$ - the velocity in $\mathrm{m} / \mathrm{s}$ and $p$ - the pressure in $\mathrm{Pa}$.

The model utilizes the Lagrangian acceleration referential bound to the ball, the density of the volume force is $\boldsymbol{F}$ and its radial and vertical components are, respectively

$$
\begin{aligned}
& F_{r}=0, \\
& F_{z}=-\rho(a+g),
\end{aligned}
$$

where $g$ is the earth gravitation and $a$ - the acceleration of the ball in the fluid.

The differential equation of movement is

$$
m \ddot{x}=F_{\mathrm{g}}+F_{z} \text {, }
$$

where $F_{\mathrm{g}}$ is the gravitational force and $F_{z}$ is the $z$ component of the force which the water exerts on the ball.

Because the set of the balls used in the experimentation comport a ball which has a rough surface, we have remarked that its downward movement have a vortex (rotation) behavior, that is why a correction factor based on rotational term where we deduct the velocity bias has been implemented in the equation above (1) as follows:

$$
\boldsymbol{\omega}=-\operatorname{rot}(\boldsymbol{v}) \text {. }
$$

$\omega$ is the rotation of the ball due to the roughness observed during the ball falling in the experiment. The roughness factor $R_{\mathrm{g}}$ as we will see below is determinant for the accuracy of the results since it affects the viscous friction coefficient $k$ and thus the viscous friction force $F_{\mathrm{fr}}$. The forces balance acting on the ball has as component the friction force which is a resistant force and is given by

$$
F_{\text {fr }}=-k \eta \boldsymbol{v} \text {. }
$$

This correction improves considerably the accuracy of viscosity calculation, in our case it was improved about twenty times [7]. For every experiment, video scenes of ball falling within the tube in "avi" format are taken then processed. Because the sizes of videos are important (about $10 \mathrm{MB}$ ), it takes much CPU running time and thus takes wide memory size, that is why compression has been necessary by utilization of an appropriate software called Videolab $(\mathbb{R})$ integrated within $\mathrm{C}++$ language. That allows the compression of the video sequences until one hundred times, their loading and processing in the software became therefore more easy and more fast. The captures of images by step of 30 frames per second (fps) was by great utility for computing the fall height (distance) $l$.

The ball in its falling is tracked image by image, gotten an image every laps of time $t_{\mathrm{i}}$ used to compute the instantaneous velocity $v_{\mathrm{i}}$, a test is implemented in the code source in order to know if the movement reached its uniform phase (steady state regime), at this moment the terminal velocity $v_{L}$ is equivalent to the instantaneous velocity computed as the ratio of the browsed distance $l$ and the time $t_{\mathrm{i}}$ (flowchart in Fig. 1). The whole algorithm will be detailed in ulterior paragraph by flowchart implemented in the program. The cylinder tube is made of glass, its radius $R$ is equal to $15 \mathrm{~mm}$ and the balls have varying radius $r_{\mathrm{b}}$. In Stokes formula the ratio $r / R$ is an important parameter which affects the values of viscosities.

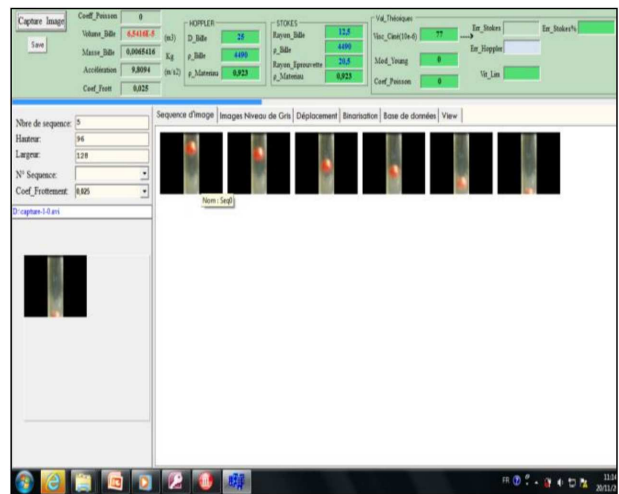

Fig. 1. General view of the software in execution, the video sequence in the left and the images binarisation in the right of the interface, above the computation results (terminal velocity, viscosities and errors between optical and experimental viscosities).

In the software interface elaborated by the way in $\mathrm{C}++$ language, we can choose parameters of experiment which are the fluid nature (density), the friction factor, the material of ball and its radius and the temperature from the Access $\mathbb{B}$ integrated database.
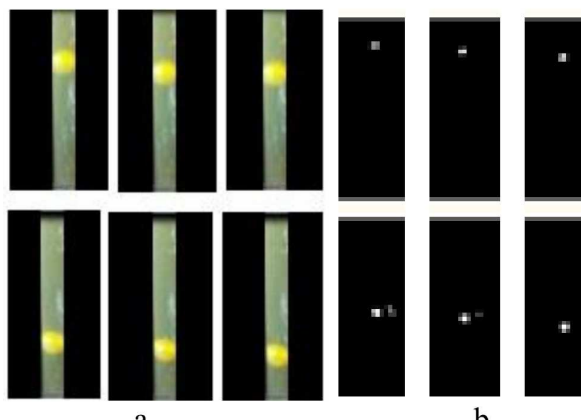

$\mathrm{b}$

Fig. 2. (a) Images capture from the video sequences of the ball falling where the distance is the difference between position in the first and the last image; (b) binarisation of images and ball tracking during its fall in the cylinder. 


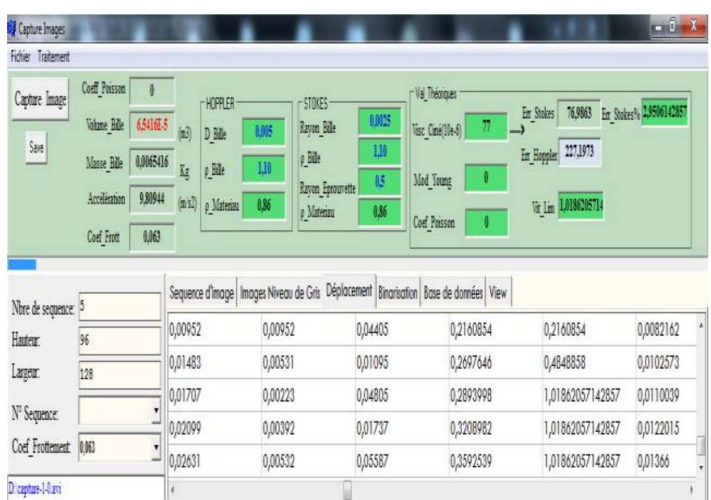

Fig. 3. Snapshot from the software where the velocities and viscosities are computed.

All video sequences are listed in directory and linked to the parameters choices in such way that every sequence is loaded relevant to the choice as it can be seen in Figs. 2 and $3[8]$.

\section{Results}

Combination of all parameters cited above gave results hereafter, and thus allows us to get a precise idea about the most adequate condition for a precise determination of fluid's dynamic viscosity by optical method (determined by video capture of ball movement) [9]. To push the study to its high level, we compare the optical values with viscosities given by a series of manipulations on the Couette viscometer.

\subsection{Terminal velocity in function of fluid density}

As we see in the snapshot below, the density of fluid affects considerably the terminal velocity, for example for the vegetal oil which has the least density, the velocity is equal to $1.2 \mathrm{~m} / \mathrm{s}$ for the test using the steel ball with diameter of $17 \mathrm{~mm}$, this value is the highest value for the experiments performed in this work.

The fluid with lowest viscosity (vegetal oil in blue line) has the highest terminal velocity as we can see in the graph above; contrarily, in the industrial high viscosity oil SAE140, this velocity increases, since the viscous force acts negatively on the movement and tends to brake it [10]. The asperity present in the curves denotes that the ball undergoes a fluctuation in its movement due to the vortices movement induced by the roughness (phenomena observed in the experiments), that is why we have introduced the correction term cited above in Eq. (6).

The highest terminal velocity is reached for vegetal oil using the steel ball for the highest temperature of $98.6^{\circ} \mathrm{C}$ which mean in this case that the viscosity is lowest possible, because all parameters (weak roughness, high weight) [11] are gathered for fast fall of the ball as is shown by dashed line in the above chart (Fig. 4). In physical sense, the velocity would be low in laminar flow of fluid and high if the flow is turbulent on condition it meets no obstacle, which is not the case in our experiments.

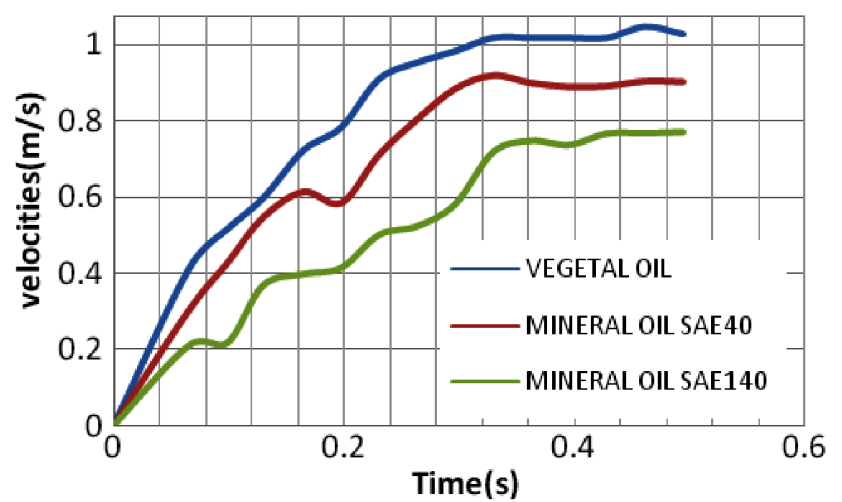

Fig. 4. Terminal velocities in function of different viscosities of fluids at $98.6^{\circ} \mathrm{C}$ (steel ball diameter equal to $25 \mathrm{~mm})$.

We denote also that we cannot interminably increase the diameter of the ball in order to obtain fast fall like it has been explained previously, due to Archimedes thrust.

\subsection{Influence of the ball roughness on terminal velocity}

The graphs below (Fig. 5) show that the velocity is inversely proportional (decreases with) to the roughness and proportional (increases with) to the diameter. For example, the velocity is about $1.02 \mathrm{~m} / \mathrm{s}$ for a roughness $R_{\mathrm{g}}=0.063$ [2], diameter $D=25 \mathrm{~mm}$, because if the diameter increases, obviously the volume and the weight of the ball increase with, so that the force of gravitation gives to the fall a high velocity [12].
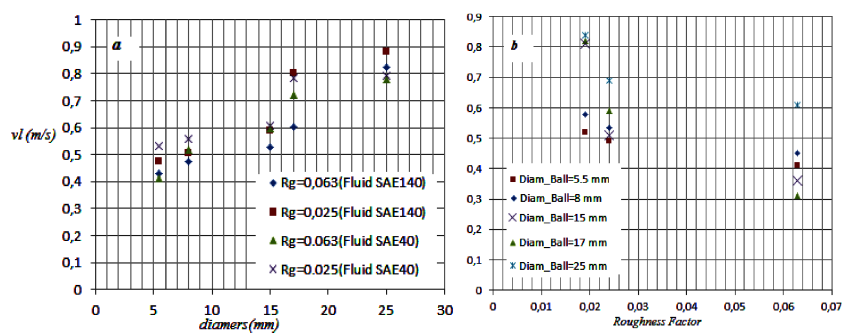

Fig. 5. Effect of roughness on the terminal velocity $v_{l}$ in function of diameter variation for different fluids (SAE140, SAE40): (a) for fixed roughness factors; (b) for fixed diameters.

However, this augmentation cannot be indefinite, since the high diameter increases the contact surface and also the thrust of Archimedes [3] which is a resistant force and thus decreases the velocity. Contrarily, the roughnesses of the ball surface mean more contact surface between the ball and the fluid, thus the resistant friction force increases and decreases the velocity [13].

\subsection{The viscosity accuracy}

Hereafter, we present an exhaustive comparison between the dynamic viscosities computed by optical 
method and those given by manipulation of Couette viscometer cited in the previous paragraph. The comparison is carried out by computing relative difference as following:

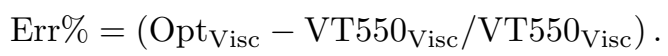

By changing the parameters of the calculation in the software, the different obtained errors vary in the best case between $0.039 \%$ and $2.81 \%$ (uncertainty equal $0.5 \%$ to $2.0 \%$ for the commercial ball fall viscometer) [2]. That denotes that we have obtained good accuracies due essentially to an implementation of a robust algorithm in calculation.

\subsection{Representation of errors between optical and standard data}

As it is shown in the graph below, for a high viscous fluid (Fig. 6) the ball material which is most adequate for optical measurement is the steel in condition that a low roughness surface is a characteristic of the material [14]. This adequation is verified only if the ball has an adequate diameter $(15.5-25.5 \mathrm{~mm})$ because when diameter increases the weight is increasing with. These parameters optimization allows the elimination of the bias which blemishes the measurement results [15]. If we take in
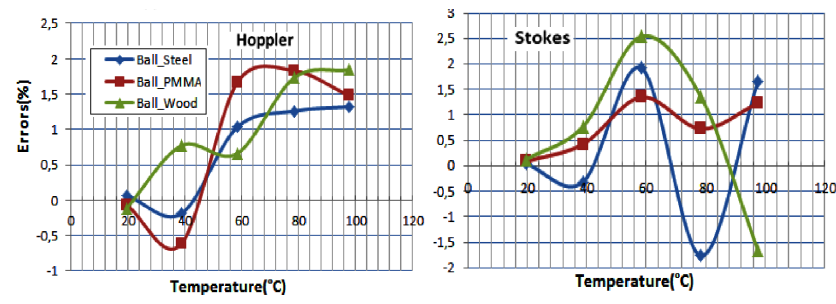

Fig. 6. Temperatures dependences of the relative error of high viscosity fluid (SAE140) in fuction of ball material for a fixed diameter $D=5.5 \mathrm{~mm}$. Both methods of Stokes and Hoppler.
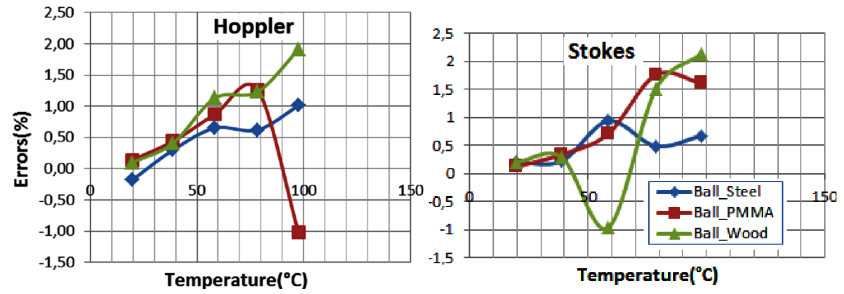

Fig. 7. Temperature variation of the relative error of middle viscosity fluid (SAE 40) for different ball material and fixed diameters $(5.5 \mathrm{~mm}, 8 \mathrm{~mm})$, both methods of Stokes and Hoppler.

consideration non-optimized conditions in optical experimentation performed in this work, such as a weak speed of camera which is about 30 frames per second and also bad condition of lightening, we can consider that we have got satisfying results of optical measurement [16]. Figures 7-9 illustrate clearly these assumptions.
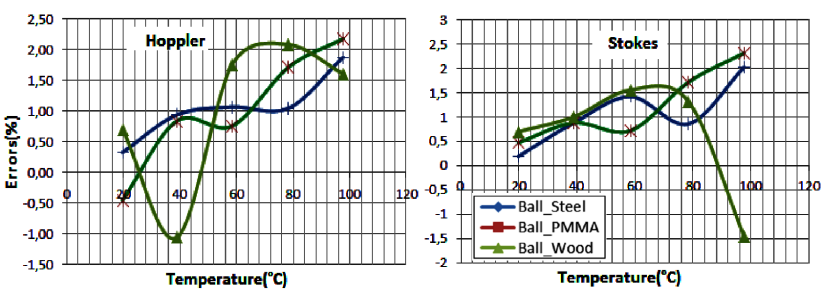

Fig. 8. Temperature variation of the relative error of low viscosity fluid (vegetal oil HVEG) for different ball material and fixed diameters $(5.5 \mathrm{~mm}, 8 \mathrm{~mm})$, both methods of Stokes and Hoppler.
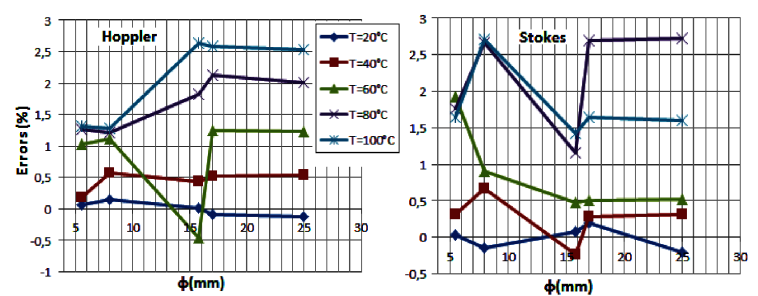

Fig. 9. Variation of uncertainties relevant to variation of diameters, for different temperatures (Hoppler and Stokes methods) fluid with high viscosity (SAE140).

\section{Discussion}

Relatively to this study, we can conclude the following results:

- The best values for relative uncertainty are given by the most viscous fluid SAE140. - That is due to the relative low speed of the fall which allows the optical system to take picture adequately. - The roughness of the balls has been voluntarily introduced in order to decrease the terminal velocity of the fall so that allow a good video tracking of the movement in the fluid.

- Good time estimation of the fall is an important parameter for viscosity calculation.

- The experimentation shows that roughness has a strong influence on the accuracy of the rheological characterization of the fluids, for example when the roughness factor is equal to 0.063 the terminal velocity does not exceed $0.75 \mathrm{~m} \mathrm{~s}^{-1}$ in all cases (steel, PMMA and wood material) so that the accuracy reaches good values approaching an incertitude of 0.009 .

Globally, we can consider the optical method regarding the experimentation conditions, which has done its proof relevant to the measures incertitude over the height of fall and terminal velocity. It is proved also that combination of optimized parameters has improved the accuracy and confirms the efficiency of this technique.

Finally, it is clear that this method despite its good results, has its limit and its dependences on a drastic choice of experimental parameters and rigorous use of mathematical formalism which govern the flow and viscosity assessment, task which is not always obvious, but at least it remains a lot to do in order to improve an innovative method for physical parameters measurements. 


\section{References}

[1] M. Fowler, Viscosity Introduction: Friction at the Molecular Level, Lectures on Fluids, University of Virginia Publ., 2007.

[2] Comsol, Terminal Falling Velocity of a Sand Grain Solved with Comsol Multiphysics 3.5a, Comsol Multiphysics Software Demonstration, 2010.

[3] S. Gillet, Viscosity: Elementary Course, Académie des Sciences (France), Elsevier Masson, 2008 (in French).

[4] www.wikipedia.org/wiki/Masse volumique des matériaux (Volume Mass of Materials).

[5] N. Ashrafi, Appl. Rheol. 22, 34203 (2012).

[6] J. Chaurette, Fiberglass Pipe Handbook, Design Incorporation, SPI Composites Institute, Montreal 2003.

[7] A. Marcinkowska-Gapinska, P. Kowal, Acta Phys. Pol. A 125, A-24 (2014).

[8] M.A. Sutton, W.J. Wolters, W.H. Peters, W.F. Ranson, S.R. McNeil, Image Vis. Comput. 1, 133 (1983).

[9] Instruction Manual, Description of the function elements and measuring ranges of the measuring instrument, for the Viscotester VT550 (2009).
[10] A. Lefebvre, B. Maury, Apparent Viscosity of a mixture of a Newtonian Fluid and Interacting Particles, Laboratoire de Mathématiques, Université Paris-Sud, 2005.

[11] A. Kushima, X. Lin, J. Li, X.F. Qian, J. Eapen, J.C. Mauro, P. Diep, S. Yip, J. Chem. Phys. 131, 164505 (2009).

[12] F.B. Smith, F.G. Fowkes, A. Rumley, A.J. Lee, Eur. Heart J. 14, 597 (1993).

[13] J.D. Tice, A.D. Lyon, R.F. Ismagilov, Anal. Chim. Acta 504, 73 (2004).

[14] E. Szwajczak, R. Stragaczyński, H. Herba, J. Jadżyn, Acta Phys. Pol. A 115, 905 (2009).

[15] T. Schweizer, Appl. Rheol. 14, 197 (2004).

[16] J. Russ, The Image Processing Handbook, 6th ed., North Carolina State University Materials Science and Engineering, CRC Press is an imprint of the Taylor and Francis Group, 2011. 